\title{
Sinusoidal Obstruction Syndrome and Postoperative Complications Resulting from Preoperative Chemotherapy for Colorectal Cancer Liver Metastasis
}

\author{
TORU HISAKA ${ }^{1}$, HIROTO ISHIKAWA ${ }^{1}$, HISAMUNE SAKAI ${ }^{1}$, RYUICHI KAWAHARA ${ }^{1}$, \\ YUICHI GOTO ${ }^{1}$, YORIKO NOMURA $^{1}$, MASAFUMI YASUNAGA ${ }^{1}$, TETSUSHI KINUGASA ${ }^{1}$, \\ FUMIHIKO FUJITA ${ }^{1}$, TOMOAKI MIZOBE ${ }^{1}$, MASARU FUKAHORI ${ }^{2}$, KEISUKE MIWA ${ }^{2}$, \\ OSAMU NAKASHIMA ${ }^{3}$, MASAHIKO TANIGAWA ${ }^{4}$, YOSHIKI NAITO ${ }^{4}$, JUN AKIBA ${ }^{4}$, \\ HIROHISA YANO $^{5}$, HIROYUKI TANAKA ${ }^{1}$, YOSHITO AKAGI ${ }^{1}$ and KOJI OKUDA ${ }^{1}$ \\ Departments of ${ }^{1}$ Surgery and ${ }^{5}$ Pathology, Faculty of Medicine, Kurume University, Kurume, Japan; \\ ${ }^{2}$ Multidisciplinary Treatment Cancer Center, ${ }^{3}$ Department of Clinical Laboratory Medicine and \\ ${ }^{4}$ Department of Diagnostic Pathology, Kurume University Hospital, Kurume, Japan
}

\begin{abstract}
Background/Aim: The aim of this study was to investigate the effects of preoperative chemotherapy on the healthy, metastasis-free part of the liver in colorectal cancer patients with liver metastasis, and the relationship between chemotherapy and postoperative complications. Patients and Methods: Our study included 90 cases of colorectal cancer liver metastasis resected after preoperative chemotherapy. The patients were divided into three groups according to the received chemotherapy regimen: 20 cases received mFOLFOX6, 54 cases a combination of mFOLFOX6 with bevacizumab, and 16 cases a combination of MFOLFOX6 and cetuximab or panitumumab. Results: The mean numbers of sinusoidal injuries for each chemotherapy type were compared. The group treated with the combination of MFOLFOX6 and bevacizumab showed a lower extent of sinusoidal injury relative to other groups; this intergroup difference became increasingly remarkable as the number of chemotherapy cycles increased. Complications of various extents were found in all three groups, but no significant differences were observed between the three groups. Conclusion: In cases where preoperative chemotherapy was extended over a long period, combined use of bevacizumab was thought to be effective
\end{abstract}

This article is freely accessible online.

Correspondence to: Toru Hisaka, MD, Ph.D., Department of Surgery, Faculty of Medicine, Kurume University, 67 Asahi-machi, Kurume, Fukuoka 830-0011, Japan. Tel: +81 942317566, Fax: +81 942340709, e-mail: thisaka@med.kurume-u.ac.jp

Key Words: Sinusoidal obstruction syndrome, oxaliplatin, clinicopathological study, postoperative complications. because of stabilization of disturbed liver hemodynamics resulting from sinusoidal injury suppression effects, allowing effective distribution of anti-cancer agents to tumors.

Colorectal cancer is one of the most frequently occurring malignant tumors worldwide (1). Approximately $60 \%$ of colon cancer patients have been reported to have complications of liver metastasis during the course of their disease (2). Surgical treatment presents the only possibility of curative treatment for colorectal cancer liver metastasis and has a strong impact on prognosis. A variety of efforts in recent years including preoperative liver function evaluation, preoperative chemotherapy, surgical procedures, preoperative management, and so on, have been reported to improve prognosis and liver resectability. Still, the major factor is the burden of chemotherapy itself (3-7). It has been reported (8) that, in cases where radical resection is possible, colorectal cancer liver metastasis shows approximately $60 \%$ recurrence, $80 \%$ of which are unresectable. Thus, chemotherapy will certainly play an increasingly important role in the future.

Preoperative chemotherapy for colorectal cancer liver metastasis plays an important role in a regimen of multidisciplinary treatment. Chemotherapy also involves tissue adverse effects, and there are apprehensions regarding the effects on remnant liver when hepatectomy is performed following systemic chemotherapy (9). Veno-occlusive disease (VOD) (10), also known as sinusoidal obstruction syndrome (SOS), is a pathology that causes injury to sinusoidal and venule endothelial cells and is reported to be caused by radiotherapy, chemotherapy, and so on. Numerous reports have stated that SOS incidence is high in chemotherapy for colorectal cancer liver metastasis, especially in regimens that include oxaliplatin (11). Severe post-operative complications thought to occur due 
to SOS have also been reported (12), thus necessitating even greater caution when performing chemotherapy. Thus, as chemotherapy becomes increasingly important for colorectal cancer liver metastasis, simultaneous assessment of liver damage is required for increasing the safety of multidisciplinary treatment of this metastasis. This study aimed to clarify the relationship between preoperative chemotherapy on the healthy metastasis-free liver and postoperative complications.

\section{Patients and Methods}

This study included 90 cases of colorectal cancer liver metastasis resected after administration of preoperative chemotherapy at Kurume University Hospital and Kurume University Medical Center from 2008 to 2015. Patients were classified into three groups, according to preoperative chemotherapy as follows: mFOLFOX6, after bolus injection of $400 \mathrm{mg} / \mathrm{m}^{2} 5$-FU, intravenous infusion of $85 \mathrm{mg} / \mathrm{m}^{2}$ oxaliplatin, and $200 \mathrm{mg} / \mathrm{m}^{2} \mathrm{dl}$ leucovorin, followed by $2400 \mathrm{mg} / \mathrm{m}^{2}$ 5-FU, over $46 \mathrm{~h}$ administration; mFOLFOX6 combined with 5 $\mathrm{mg} / \mathrm{kg}$ bevacizumab; mFOLFOX6 combined with cetuximab initially at $400 \mathrm{mg} / \mathrm{m}^{2}$ followed by $250 \mathrm{mg} / \mathrm{m}^{2}$, or combined with 6 $\mathrm{mg} / \mathrm{kg}$ panitumumab. Patients with the combination including bevacizumab were treated with only mFOLFOX6 as their final chemotherapy regimen. Hepatectomy of patients with the combination treatment including bevacizumab was performed 6 to 9 weeks after the final treatment day, whereas hepatectomy of patients with the combination treatment including cetuximab or panitumumab was performed 4 to 7 weeks after the final treatment day. ClavienDindo Classification (13) was used to assess the postoperative complications related to hepatectomy. After fixing the excised specimens in $10 \%$ neutral buffered formalin solution, sections were created from sites located at $3 \mathrm{~cm}$ or further away from the tumor, and hematoxylin-eosin (HE) staining, Masson trichrome staining, and silver impregnation staining were performed. Microscopic assessment coupled with immunostaining was performed as described below.

Immunohistochemistry. We used 4- $\mu$ m-thick sections of formalin-fixed, paraffin embedded tissues. The sections were mounted on glass slides and immunocytochemistry with anti-mouse monoclonal antibody against human CD34 (clone QBEnd/10, IgG1, no dilutio ; Leica Biosystems Newcastle, Ltd., Newcastle, UK) and anti-mouse monoclonal antibody against human smooth muscle actin (clone $\alpha \mathrm{sm} 1$, IgG2a, no dilution; Leica Biosystems) was performed on the same fully automated Bond-III system (Leica Microsystems) using onboard heatinduced antigen retrieval with epitope retrieval solution 2 for $20 \mathrm{~min}$ at $99^{\circ} \mathrm{C}$. Each slide was incubated with the antibody for $30 \mathrm{~min}$ at room temperature. This automated system uses a Refine polymer detection kit with horseradish peroxidase-polymer as a secondary antibody and DAB. Incubation with a secondary antibody was performed for $30 \mathrm{~min}$ at room temperature. All immunohistochemical analyses were evaluated by three experienced observers who were unaware of the patients' conditions.

SOS evaluation. Our sinusoidal injury assessment method involved random extraction of 10 visual fields at $40 \times$ magnification, while ensuring that these fields were sufficiently distant from the tumor, and calculation of the mean number of sinusoidal injuries per visual field. After observation of HE-stained specimens at sites determined as having sinusoidal injury, the same specimens were used for silver
Table I. Clinical characteristics of patients with CRLM.

\begin{tabular}{lc}
\hline Clinical characteristic & Number of patients \\
\hline Age (years) & \\
Range & $36-82$ \\
Median & 61 \\
Gender & \\
Male & 56 \\
Female & 34 \\
Primary tumor & \\
Colon & 52 \\
Rectum & 38 \\
Lymph nodes metastasis & \\
Absent & 28 \\
Present & 62 \\
Timing of metastasis & \\
Metachronous & 42 \\
Synchronous & 48 \\
Liver metastasis & \\
Solitary & 30 \\
Multiple & 60 \\
Tumor size & \\
$<5 \mathrm{~cm}$ & 75 \\
$\geq 5 \mathrm{~cm}$ & 15 \\
\hline
\end{tabular}

impregnation staining, masson trichrome staining, and immunostaining for $\alpha$-smooth muscle actin and CD34. Sinusoidal injury evaluation was performed by three pathologists.

Statistical analysis. Student's $t$-test or the chi-square test was used to evaluate differences between categorical variables. The KruskalWallis test was used for quantitative variables in three or more groups. Significance level was set at $p<0.05$, and JMP Pro 11.0.0 (SAS Institute Inc., Cary, NC, USA) was used for the analysis.

\section{Results}

A total of 90 cases of hepatectomy performed after administering chemotherapy were included in this study and patient ages ranged from 36 to 82 years and the mean age was 61 years. The primary tumor was in the colon in 52 cases and in the rectum in 38 cases, with a majority confirmed to be accompanied by lymph node metastasis. There were 42 cases with metachronous liver metastasis, and 48 cases with synchronous liver metastasis. There were 30 cases of solitary liver metastasis and 60 cases with multiple liver metastasis. As for tumor diameter, 75 cases had tumors of less than $5 \mathrm{~cm}$, and 15 cases had a diameter of $5 \mathrm{~cm}$ or above (Table I).

Chemotherapy breakdown was as follows: 20 cases were administered mFOLFOX6, 54 cases were administered a combination of mFOLFOX6 with bevacizumab, and 16 cases were treated with a combination of mFOLFOX6 and cetuximab or panitumumab. The mFOLFOX6-only group was administered 3 to 12 cycles, with a mean of 7.2 cycles; 


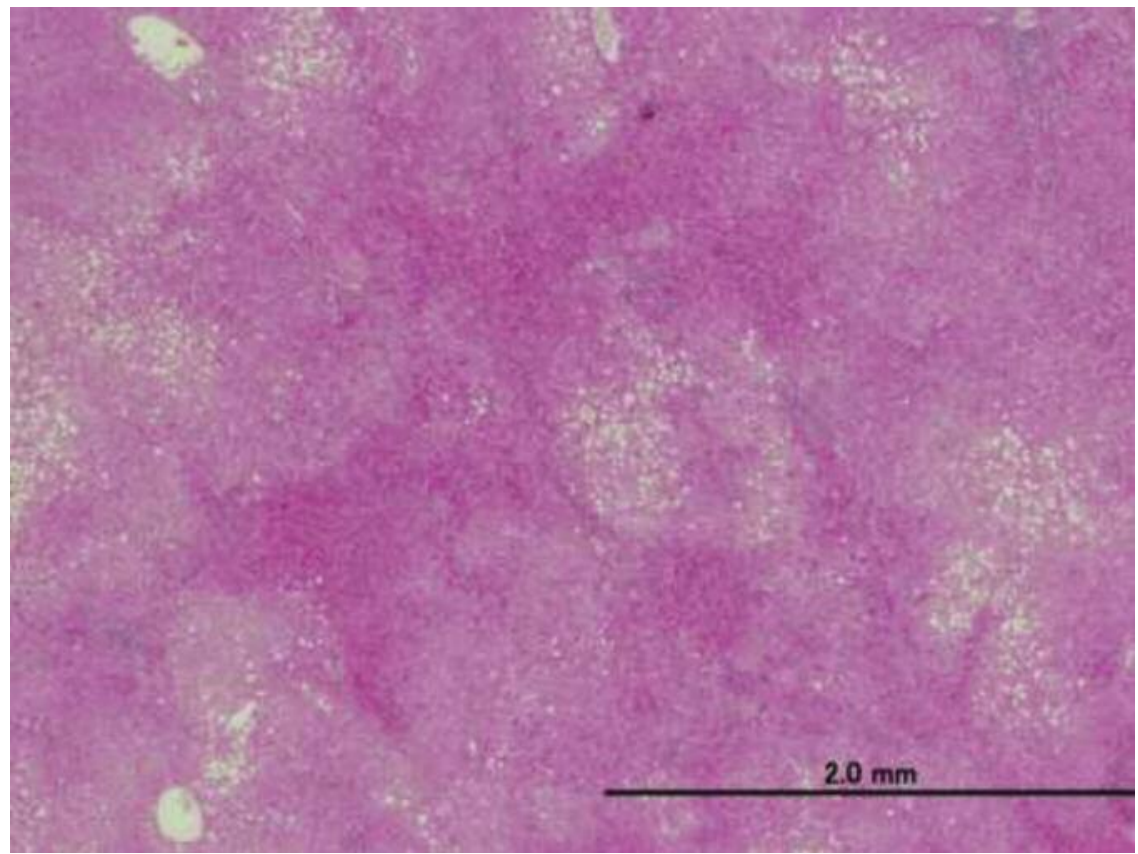

Figure 1. Photomicrograph of sinusoidal injuries. Sinusoidal dilation showed irregular distribution, reaching the neighboring hepatic lobules (HE staining, $\times 20$ ).
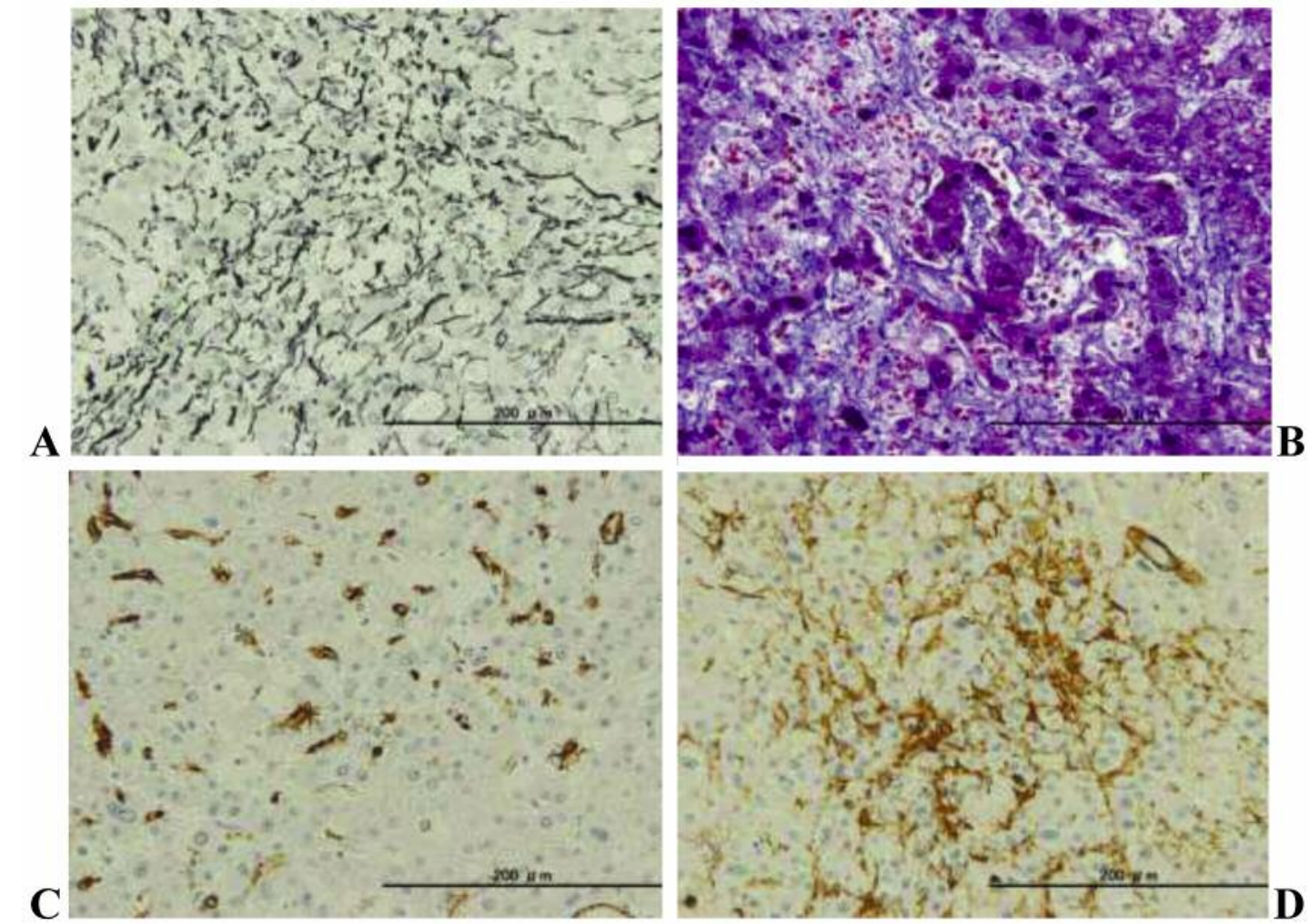

Figure 2. Photomicrographs of sinusoidal injuries. (A) Hepatic cord configuration became obscure, and reticular fiber intricacies and disruptions were observed (Silver impregnation staining, $\times 200$ ). (B) Proliferation of collagen fibers was confirmed (Masson trichrome staining, $\times 200$ ), (C) along with CD34 positive findings matching the mutated sinusoidal endothelial cells (CD34 immunostaining, $\times 200)$. (D) At the same sites, $\alpha$-SMA positive myofibroblasts were observed ( $\alpha$-SMA immunostaining, $\times 200$ ). 
Table II. Comparison of sinusoidal injuries and postoperative complications in preoperative chemotherapy.

\begin{tabular}{lccc}
\hline Regimens & mFOLFOX6 & $\begin{array}{c}\text { mFOLFOX6 } \\
\text { +Becacizumab }\end{array}$ & $\begin{array}{c}\text { mFOLFOX6 } \\
\text { +Cetuximab/Panitumumab }\end{array}$ \\
\hline Number of patients & 20 & 54 & 16 \\
Cycles & $3 \sim 12$ & $4 \sim 23$ & $4 \sim 13$ \\
$\quad$ Mean & 7.1 & 8.9 & 7.6 \\
Number of SI & $1.7 \pm 0.8$ & $0.2 \pm 0.2$ & $1.6 \pm 1.00 .0004$ \\
$\quad$ total & $0.2 \pm 0.4$ & & $0.2 \pm 0.2$ \\
Number of SI & $1.1 \pm 0.6$ & $0.06 \pm 0.1$ & $1.2 \pm 1.0$ \\
$1-5$ cycles & $1.7 \pm 0.8$ & $0.13 \pm 0.1$ & $1.6 \pm 1.0$ \\
$6-10$ cycles & 5 & $0.4 \pm 0.2$ & 4 \\
11 cycles & 5 & 21 & 2 \\
CDC & 2 & 6 & 2 \\
1 & 3 & 1 & 0 \\
2 & 0 & 0 & 0 \\
3 & 0 & 0 & 0.023 \\
4 & 0 & & 0.004 \\
\hline
\end{tabular}

FOLFOX: Chemotherapy with folinic acid, 5-fluorouracil, and oxaliplatin; SI: sinusoidal injuries; CDC: Clavien-Dindo classification.

the mFOLFOX6 with bevacizumab group was administered 4 to 23 cycles, with a mean of 8.9 cycles; the mFOLFOX6 with cetuximab or panitumumab group was administered 4 to 13 cycles, with a mean of 7.6 cycles (Table II).

Clinical images of excised liver were used to determine whether histological sinusoidal endothelial cell injury had occurred due to preoperative chemotherapy. Histologically, sinusoidal dilation showed irregular distribution, reaching the neighboring hepatic lobules (Figure 1). These findings emerged unrelated to individual hepatic lobule units, with a so-called "mottled" configuration. Under high-power magnification, irregular and ruptured hepatic cords were observed, with hepatocyte degeneration and atrophy. Nevertheless, there was no confirmation of hepatocyte necrosis. Upon silver impregnation staining, hepatic cord configuration became obscure, and reticular fiber intricacies and disruptions were observed (Figure 2A). Upon Masson trichrome staining, proliferation of collagen fibers was confirmed (Figure 2B), along with CD34 positive findings matching the mutated sinusoidal endothelial cells (Figure $2 \mathrm{C}$ ). At the same sites, $\alpha$-SMA positive myofibroblasts were found (Figure 2D). SOS assessment or evaluation of the extent of sinusoidal injury was then performed. The mean unit numbers of sinusoidal injury for each chemotherapy method were compared. It was found that the mFOLFOX6 combined with bevacizumab group had lower levels of sinusoidal injury compared with that in the other groups. Comparing this with the respective chemotherapy cycle numbers, though no significant differences were found among the three groups up to less than 5 cycles, the mFOLFOX6 combined with bevacizumab group showed significantly lower levels of sinusoidal injury from cycle 5 onward, and this significance became especially remarkable after 10 cycles (Table II).

Next, postoperative complications were investigated. Assessment of such cases was performed in accordance with the Clavien-Dindo Classification (13), with comparative examination among the three groups. While all three groups showed complications at various grades, no significant differences were found among groups. Complications of Clavien-Dindo Classification grade IV and above were not found in any of the groups. There were six cases judged as Clavien-Dindo Classification grade III (Table II). In the mFOLFOX6-only group, two of three cases demonstrated bile leakage, whereas there was one case of intra-abdominal infection. Large right pleural effusions were observed in the mFOLFOX6 with bevacizumab combination group. Both cases of bile leakage were observed in the mFOLFOX6 with cetuximab or panitumumab combination group. Puncture drainage was performed for all cases.

\section{Discussion}

The relationship between sinusoidal injury and chemotherapy in colon cancer, especially when oxaliplatin is used, has been previously reported (9). The present study revealed similar findings. Our sinusoidal injury assessment method involved random extraction of 10 visual fields at $40 \times$ magnification, with evaluation of the mean number per visual field. This was done for two reasons: first, these are not changes that occur at the individual hepatic lobe unit; second, these are not diffuse changes. In our investigation, 
there was no regularity in terms of occurrence at specific sites, but instead these changes were detectable at any site with endothelial cells. Figure 1 shows the injury and displacement of sinusoidal endothelial cells, congestion and fibrosis within dilated sinusoids, disorganized hepatic cords, and atrophied hepatocytes. Although many of such injuries occurred at increased numbers of chemotherapy cycles, no severe damage such as hepatocyte necrosis, cholestasis, etc., was found in any of the investigated cases. Therefore, it is highly possible that the effects of chemotherapy for colorectal cancer liver metastasis are mainly pathologies of local hepatic circulation injuries not accompanied by hepatocyte necrosis. In the combination using bevacizumab, sinusoidal injury was observed at a reduced extent relatively to other groups. This result is similar to those reported previously (14). It is thought that suppression of sinusoidal endothelial cell damage due to combination chemotherapy with bevacizumab will have a positive impact on chemotherapy effects. Thus, suppression of sinusoidal injury by bevacizumab helps stabilize liver hemodynamics, indicating the possibility that anti-cancer agents can be maintained at quantities effective against tumors. Therefore, for cases assumed to require large numbers of preoperative chemotherapy cycles, our results suggest that the combination treatment with bevacizumab might be effective.

The incidence of clinically relevant complications was found to be similar between the three regimens. Therefore, regarding the frequency of complications, there is likely no need for a particular concern when selecting any of the studied treatment methods. Fukuoka et al. have made a comparative study of postoperative complications regarding administration or not of preoperative chemotherapy, or regarding chemotherapy regimen. However, no clear differences were found (15). In our study, although we found no clear differences, we did recognize common factors for the six cases with Clavien-Dindo Classification of grade III or above. In all 6 cases, major hepatectomy was performed at 3 or more sectionectomies and preoperative chemotherapy had been administered 11 or more times (data not shown). Bile leakage, which is a grave complication for hepatectomy, was observed in 2 cases from the mFOLFOX6-only group, and 2 cases from the mFOLFOX6 combined with cetuximab or panitumumab group. It is, thus, thought that for cases that require long-term preoperative chemotherapy, special care is needed, particularly, in those cases where relatively major hepatectomy is required. In conclusion, our study clarified the relationships between sinusoidal injury due to preoperative chemotherapy and postoperative complications. Chemotherapy that included oxaliplatin caused sinusoidal injury, which became more remarkable with increased number of cycles of chemotherapy. However, combined use of bevacizumab suppressed such injuries. Therefore, we consider that a combination regimen with bevacizumab will be effective in cases where it is thought that preoperative chemotherapy will be performed many times, as bevacizumab stabilizes liver hemodynamics resulting by suppressing sinusoidal injury, thus allowing effective distribution of anti-cancer agents to tumors. Further, in cases where preoperative chemotherapy treatment is administered multiple times, and major hepatectomy is also required, sufficient consideration and care must be given with regard to postoperative complications.

\section{Conflicts of Interest}

The Authors declare no conflicts of interest associated with this manuscript.

\section{Authors' Contributions}

Toru Hisaka designed the study, and wrote the initial draft of the manuscript. Toru Hisaka and Hiroto Ishikawa contributed to analysis and interpretation of data, and assisted in the preparation of the manuscript. All other Authors have contributed to data collection and interpretation, and critically reviewed the manuscript. All Authors approved the final version of the manuscript, and agree to be accountable for all aspects of the work in ensuring that questions related to the accuracy or integrity of any part of the work are appropriately investigated and resolved.

\section{References}

1 Torre LA, Bray F, Siegel RL, Ferlay J, Lortet-Tieulent J and Jemal A: Global cancer statistics, 2012. CA Cancer J Clin 65: 87-108, 2015. PMID: 25651787. DOI: 10.3322/caac.21262

2 Kemeny N: The management of resectable and unresectable liver metastases from colorectal cancer. Curr Opin Oncol 22: 364-373, 2010. PMID: 20520544. DOI: 10.1097/CCO.0b 013e32833a6c8a

3 Abdalla EK, Vauthey JN, Ellis LM, Ellis V, Pollock R, Broglio KR, Hess K and Curley SA: Recurrence and outcomes following hepatic resection, radiofrequency ablation, and combined resection/ablation for colorectal liver metastases. Ann Surg 239: 818-825, 2004. PMID: 15166961. DOI: 10.1097/01.sla.000 0128305.90650.71

4 Kopetz S and Vauthey JN: Perioperative chemotherapy for resectable hepatic metastases. Lancet 371: 963-965, 2008. PMID: 18358910. DOI: 10.1016/S0140-6736(08)60429-8

5 Nordlinger B, Van Cutsem E, Gruenberger T, Glimelius B, Poston G, Rougier P, Sobrero P and Ychou M: European Colorectal Metastases Treatment Group: Combination of surgery and chemotherapy and the role of targeted agents in the treatment of patients with colorectal liver metastases: recommendations from an expert panel. Ann Oncol 20: 985-992, 2009. PMID: 19153115 . DOI: $10.1093 /$ annonc/mdn 735

6 Beppu T, Emi Y, Tokunaga S, Oki E, Shirabe K, Ueno S, Kuramoto M, Kabashima A, Takahashi I, Samura H, Eguchi S, Akagi Y, Natsugoe S, Ogata Y, Kakeji Y, Baba H and Maehara Y; Kyushu Study group of Clinical Cancer (KSCC): Liver resectability of advanced liver-limited colorectal liver metastases following mFOLFOX6 with bevacizumab (KSCC0802 Study). Anticancer Res 34: 6655-6662, 2014. PMID: 25368271. 
7 Beppu T, Hayashi N, Masuda T, Komori H, Horino K, Hayashi H, Okabe H, Baba Y, Kinoshita K, Akira C, Watanebe M, Takamori $\mathrm{H}$ and Baba $\mathrm{H}$ : FOLFOX enables high resectability and excellent prognosis for initially unresectable colorectal liver metastases. Anticancer Res 30: 1015-1020, 2010. PMID: 20393029.

8 Portier G, Elias D, Bouche O, Rougier P, Bosset JF, Saric J, Belghiti J, Piedbois P, Guimbaud R, Nordlinger B, Bugat R, Lazorthes $\mathrm{F}$ and Bedenne L: Multicenter randomized trial of adjuvant fluorouracil and folinic acid compared with surgery alone after resection of colorectal liver metastases: FFCD ACHBTH AURC 9002 trial. J Clin Oncol 24: 4976-4982, 2006. PMID: 17075115. DOI: 10.1200JCO.2006.06.8353

9 Morris-Stiff G, White AD, Gomez D, Cameron IC, Farid S, Toogood GJ, Lodge JP and Prasad KR: Nodular regenerative hyperplasia (NRH) complicating oxaliplatin chemotherapy in patients undergoing resection of colorectal liver metastases. Eur J Surg Oncol 40: 1016-1020, 2014. PMID: 24370284. DOI: 10.1016/j.ejso.2013.09.015

10 Reed GB Jr and Cox AJ Jr.: The human liver after radiation injury. A form of veno-occlusive disease. Am J Pathol 48: 597611, 1966. PMID: 5327788.

11 Rubbia-Brandt L, Audard V, Sartoretti P, Roth AD, Brezault C, Le Charpentier M, Dousset B, Morel P, Soubrane O, Chaussade S, Mentha G and Terris B: Severe hepatic sinusoidal obstruction associated with oxaliplatin-based chemotherapy in patients with metastatic colorectal cancer. Ann Oncol 15: 460-466, 2004. PMID: 14998849. DOI: 10.1093/annonc/mdh095

12 Nordlinger B, Sorbye H, Glimelius B, Poston GJ, Schlag PM, Rougier P, Bechstein WO, Primrose JN, Walpole ET, FinchJones M, Jaeck D, Mirza D, Parks RW, Collette L, Praet M, Bethe U, Van Cutsem E, Scheithauer W and Gruenberger T; EORTC Gastro-Intestinal Tract Cancer Group; Cancer Research UK; Arbeitsgruppe Lebermetastasen und-tumoren in der
Chirurgischen Arbeitsgemeinschaft Onkologie (ALM-CAO); Australasian Gastro-Intestinal Trials Group (AGITG); Fédération Francophone de Cancérologie Digestive (FFCD): Perioperative chemotherapy with FOLFOX4 and surgery versus surgery alone for resectable liver metastases from colorectal cancer (EORTC Intergroup trial 40983): a randomised controlled trial. Lancet 371(9617): 1007-1016, 2008. PMID: 18358928. DOI: 10. 1016/S0140-6736(08)60455-9

13 Dindo D, Demartines N and Clavien PA: Classification of surgical complications: a new proposal with evaluation in a cohort of 6336 patients and results of a survey. Ann Surg 240: 205-213, 2004. PMID: 15273542. DOI: 10.01097/01. Sla. 0000133083. 54934.ae

14 Hubert C, Sempoux C, Humblet Y, van den Eynde M, Zech F, Leclercq I and Gigot JF: Sinusoidal obstruction syndrome (SOS) related to chemotherapy for colorectal liver metastases: factors predictive of severe SOS lesions and protective effect of bevacizumab. HPB (Oxford) 15: 858-864, 2013. PMID: 23458554. DOI: 10. 1111/hpb. 12047

15 Fukuoka K, Nara S, Honma Y, Kishi Y, Esaki M and Shimada $\mathrm{K}$ : Hepatectomy for colorectal cancer liver metastases in the era of modern preoperative chemotherapy: Evaluation of Postoperative Complications. World J Surg 41: 1073-1081, 2017. PMID: 27679508. DOI: 10. 1007/s00268-016-3724-6

Received May 23, 2019

Revised June 17, 2019

Accepted June 18, 2019 